Dept. of Math. University of Oslo
Pure Mathematics

$\begin{array}{lr}\text { PuRe Mathematics } & \text { No. } 27 \\ \text { ISSN 0806-2439 } & \text { SePtember 2005 }\end{array}$

\title{
Optimal stochastic impulse control with delayed reaction
}

\author{
Bernt Øksendal ${ }^{1), 2)} \quad$ Agnès Sulem ${ }^{3)}$
}

November 15, 2007

\begin{abstract}
We study impulse control problems of jump diffusions with delayed reaction. This means that there is a delay $\delta>0$ between the time when a decision for intervention is taken and the time when the intervention is actually carried out. We show that under certain conditions this problem can be transformed into a sequence of iterated no-delay optimal stopping problems and there is an explicit relation between the solutions of these two problems. The results are illustrated by an example where the problem is to find the optimal times to increase the production capacity of a firm, assuming that there are transaction costs with each new order and the increase takes place $\delta$ time units after the (irreversible) order has been placed.
\end{abstract}

AMS 2000 Subject Classification: Primary 93E20, Secondary 60HXX. Key words and phases: Impulse control, jump diffusions, delayed reaction.

1) Center of Mathematics for Applications (CMA), Dept. of Mathematics, University of Oslo, Box 1053 Blindern, N-0316 Oslo, Norway, Email: oksendal@math.uio.no

2) Norwegian School of Economics and Business Administration, Helleveien 30, N-5045 Bergen, Norway

3) INRIA, Domaine de Voluceau, Rocquencourt, B.P. 105, F-78153 Le Chesnay Cedex, France, Email: agnes.sulem@inria.fr 


\section{Introduction}

Suppose that - if there are no interventions - the state $Y(t) \in \mathbb{R}^{k}$ is described by a stochastic differential equation driven by a Brownian motion $B(t) \in \mathbb{R}^{m}$ and the compensated Poisson random measure $\tilde{N}(\cdot, \cdot)=\tilde{N}_{1}(\cdot, \cdot), \ldots, \tilde{N}_{m}(\cdot, \cdot)$ of an $m$-dimensional Lévy process $\eta(t)$, as follows:

$$
\begin{aligned}
& d Y(t)=b(Y(t)) d t+\sigma(Y(t)) d B(t)+\int_{\mathbb{R}^{k}} \gamma(Y(t), z) \tilde{N}(d t, d z) ; \quad t>0 \\
& Y(0)=y \in \mathbb{R}^{k}
\end{aligned}
$$

Here $b: \mathbb{R}^{k} \rightarrow \mathbb{R}, \sigma: \mathbb{R}^{k} \rightarrow \mathbb{R}^{k \times m}$ and $\gamma: \mathbb{R}^{k} \times \mathbb{R}^{k} \rightarrow \mathbb{R}^{k \times m}$ are given functions. We assume that $B(t)$ and $\eta(t)$ are defined on a filtered probability space $\left(\Omega, \mathcal{F},\{\mathcal{F}\}_{t \geq 0}, P\right)$.

An impulse control on the process $Y(\cdot)$ is a double sequence

$$
v=\left(\tau_{1}, \tau_{2}, \ldots ; \zeta_{1}, \zeta_{2}, \ldots\right)
$$

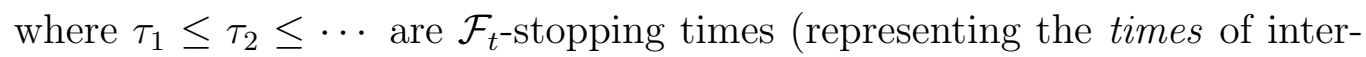
vention) and $\zeta_{1}, \zeta_{2}, \ldots$ are the corresponding intervention sizes. We assume that $\zeta_{i} \in \mathcal{Z}$, a given set, and that

$$
\zeta_{i}=\zeta_{i}(\omega) \text { is } \mathcal{F}_{\tau_{i}} \text {-measurable. }
$$

If the impulse control $v$ is applied to the process $Y(\cdot)$ we assume that the resulting process $Y^{(v)}(t)$ gets the following dynamics:

$$
\left\{\begin{array}{c}
d Y^{(v)}(t)=b\left(Y^{(v)}(t)\right) d t+\sigma\left(Y^{(v)}(t)\right) d B(t)+\int_{\mathbb{R}^{k}} \gamma\left(Y^{(v)}(t), z\right) \tilde{N}(d t, d z) \\
\tau_{j} \leq t<\tau_{j+1} \\
Y^{(v)}\left(\tau_{j+1}\right)=\Gamma\left(\check{Y}^{(v)}\left(\tau_{j+1}^{-}\right), \zeta_{j}\right) ; \quad j=1,2, \ldots
\end{array}\right.
$$

where

$$
\Gamma: \mathbb{R}^{k} \times \mathcal{Z} \rightarrow \mathbb{R}
$$

is a given function, describing the state immediately after the time $\tau_{j+1}$ of the intervention, as a function of the state right before, $\check{Y}\left(\tau_{j+1}^{-}\right)$, and the size $\zeta_{j}$ of the intervention. Here

$$
\check{Y}^{(v)}\left(\tau_{j+1}^{-}\right):=Y^{(v)}\left(\tau_{j+1}^{-}\right)+\Delta_{N} Y\left(\tau_{j+1}\right)
$$


where $\Delta_{N} Y\left(\tau_{j+1}\right)$ is the (possible) jump of $Y$ due to the jump measure $N(\cdot, \cdot)$ of $\eta$ only. The performance functional associated to the impulse control $v=\left(\tau_{1}, \tau_{2}, \ldots ; \zeta_{1}, \zeta_{2}, \ldots\right)$ is assumed to have the form

$$
J^{(v)}(y)=E^{y}\left[\int_{0}^{\tau_{s}} f\left(Y^{(v)}(t)\right) d t+g\left(Y^{(v)}\left(\tau_{S}\right)\right)+\sum_{j=1}^{N} K\left(\check{Y}^{(v)}\left(\tau_{j}^{-}\right), \zeta_{j}\right)\right]
$$

where $f: \mathbb{R}^{k} \rightarrow \mathbb{R}, g: \mathbb{R}^{k} \rightarrow \mathbb{R}$ and $K: \mathbb{R}^{k} \times \mathcal{Z} \rightarrow \mathbb{R}$ are given functions, $N \leq \infty$ is the number of interventions and

$$
\tau_{\mathcal{S}}=\inf \left\{t>0 ; Y^{(v)}(t) \notin \mathcal{S}\right\} \quad \text { (the bankruptcy time) }
$$

where $\mathcal{S} \subset \mathbb{R}^{k}$ is a given open set (the solvency region). The functions $f, g$ and $K$ represent the profit rate, terminal payoff and payoff due to intervention, respectively. We interpret $g\left(Y^{(v)}\left(\tau_{\mathcal{S}}\right)\right)$ as 0 if $\tau_{\mathcal{S}}=\infty$.

We call the impulse control $v$ admissible and write $v \in \mathcal{V}$ if

$$
E^{y}\left[\int_{0}^{\tau_{\mathcal{S}}}\left|f\left(Y^{(v)}(t)\right)\right| d t+\left|g\left(Y^{(v)}\left(\tau_{\mathcal{S}}\right)\right)\right|+\sum_{j=1}^{N}\left|K\left(\check{Y}^{(v)}\left(\tau_{j}^{-}\right), \zeta_{j}\right)\right|\right]<\infty .
$$

The classical impulse control problem is the following:

Problem 1.1 Find $\Phi(y)$ and $v^{*} \in \mathcal{V}$ such that

$$
\Phi(y)=\sup _{v \in \mathcal{V}} J^{(v)}(y)=J^{\left(v^{*}\right)}(y) .
$$

We refer to [?] for more information about impulse control in this setting. In many situations there is a delay (or a time lag) between the stopping time $\tau$ when a decision for intervention/action is taken, and the time $\tau+\delta$ when this action is carried out. Here $\delta>0$ is a constant. For example, if a shipping company decides to order a new ship, it may take a couple of years before the ship is actually delivered. In this situation the impulse control gets the form

$$
v_{\delta}=\left(\tau_{1}+\delta, \tau_{2}+\delta, \ldots ; \zeta_{1}, \zeta_{2}, \ldots\right)
$$

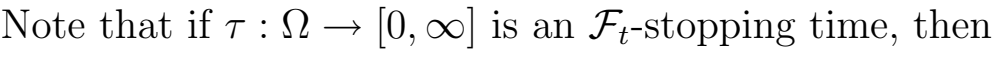

$$
\alpha:=\tau+\delta: \Omega \rightarrow[\delta, \infty]
$$


is an $\mathcal{F}_{t-\delta^{-}}$-stopping time (and hence in particular an $\mathcal{F}_{t}$-stopping time also). Indeed, we have

$$
\{\omega ; \alpha(\omega) \leq t\}=\{\omega ; \tau(\omega)+\delta \leq t\}=\{\omega ; \tau(\omega) \leq t-\delta\} \in \mathcal{F}_{t-\delta}
$$

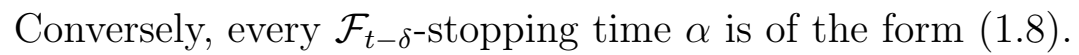

We let $\mathcal{T}_{\delta}$ denote the set of all $\mathcal{F}_{t-\delta}$-stopping times $\alpha$. In particular, $\mathcal{T}_{0}$ is the set of classical $\mathcal{F}_{t}$-stopping times $\tau$. Now define

$$
\mathcal{V}_{\delta}=\left\{v_{\delta}:=\left(\tau_{1}+\delta, \tau_{2}+\delta, \ldots ; \zeta_{1}, \zeta_{2}, \ldots\right) ; v:=\left(\tau_{1}, \tau_{2}, \ldots ; \zeta_{1}, \zeta_{2}, \ldots\right) \in \mathcal{V}\right\}
$$

For $v_{\delta} \in \mathcal{V}_{\delta}$ define

$$
\begin{aligned}
J_{\delta}^{\left(v_{\delta}\right)}(y)=E^{y} & {\left[\int_{0}^{\tau_{\mathcal{S}}+\delta} f\left(Y^{\left(v_{\delta}\right)}(t)\right) d t+g\left(Y^{\left(v_{\delta}\right)}\left(\tau_{\mathcal{S}}+\delta\right)\right)\right.} \\
& \left.+\sum_{j=1}^{N} K\left(\check{Y}^{\left(v_{\delta}\right)}\left(\left(\tau_{j}+\delta\right)^{-}\right), \zeta_{j}\right)\right]
\end{aligned}
$$

where we put $\tau_{\mathcal{S}}+\delta=\infty$ if $\tau_{\mathcal{S}}=\infty$.

In this paper we study the following delayed reaction impulse control problem:

Problem 1.2 Find $\Phi_{\delta}(y)$ and $v_{\delta}^{*} \in \mathcal{V}_{\delta}$ such that

$$
\Phi_{\delta}(y)=\sup _{v_{\delta} \in \mathcal{V}_{\delta}} J_{\delta}^{\left(v_{\delta}\right)}(y)=J_{\delta}^{\left(v_{\delta}^{*}\right)}(y)
$$

We are also interested in this problem under the constraint that at most $n$ interventions are allowed. Thus we define

$$
\mathcal{V}_{\delta}^{(n)}=\left\{v_{\delta}=\left(\tau_{1}+\delta, \tau_{2}+\delta, \ldots, \tau_{m}+\delta ; \zeta_{1}, \zeta_{2}, \ldots, \zeta_{m}\right) \in \mathcal{V}_{\delta} ; m \leq n\right\}
$$

and the $n$-intervention problem is:

Problem 1.3 Find $\Phi_{\delta}^{(n)}(y)$ and $v_{\delta}^{*} \in \mathcal{V}_{\delta}^{(n)}$ such that

$$
\Phi_{\delta}^{(n)}(y)=\sup _{v_{\delta} \in \mathcal{V}_{\delta}^{(n)}} J_{\delta}^{\left(v_{\delta}\right)}(y)=J_{\delta}^{\left(v_{\delta}^{*}\right)}(y) .
$$


It is known (see e.g. [?] and [?] that in the no-delay case $(\delta=0)$ this $n$-intervention problem can be reduced to a sequence of iterated optimal stopping problems. In Section ?? we will prove a similar result for the delayed $n$-intervention case under the assumption that $Y(\cdot)$ is $\Gamma$-homogeneous (Definition ??). In Section ?? we consider the case with no constraints on the number of interventions and in Section ?? we illustrate the result by considering a specific example.

The optimal stopping version of Problem ?? can be found in [?]. A related impulse control problem with delivery lags has been studied in [?]. Other related papers are [?] and [?]. The recent paper [?] also deals with impulse control with execution delay. That paper does not assume that $Y(\cdot)$ is $\Gamma$ homogeneous. On the other hand, it only deals with the finite horizon and diffusion case, and it assumes that the number of pending orders is uniformly bounded.

None of the above mentioned papers deal with jumps, and to the best of our knowledge our paper is the first to deal with impulse control with delayed reaction for jump diffusions. Our proofs also work in the case when there are no jumps. But the jumps do of course influence the value function and the optimal strategy, as illustrated in our example in Section ?? (Theorem ??).

\section{The case with at most $n$ interventions}

We first introduce some notation:

Definition 2.1 For $\delta \geq 0$ let $\mathcal{H}_{\delta}$ denote the set of all measurable functions $h: \mathbb{R}^{k} \rightarrow \mathbb{R}$ such that $E^{y}[|h(Y(\delta))|]<\infty$ for all $y$, where $Y(\cdot)$ is the process without intervention, given by (1.1). Then we define $\mathcal{L}: \mathcal{H}_{\delta} \rightarrow \mathcal{H}_{0}$ by

$$
\mathcal{L} h(y)=E^{y}[h(Y(\delta))] ; \quad h \in \mathcal{H}_{\delta} .
$$

We will use the following notation:

$$
\begin{aligned}
& \mathcal{L} K(y, \zeta)=E^{y}[K(Y(\delta), \zeta)] \quad \text { and } \\
& \mathcal{L}(\theta \circ \Gamma)(y, \zeta)=E^{y}[\theta(\Gamma(Y(\delta), \zeta))]
\end{aligned}
$$

if $\theta: \mathbb{R}^{k} \rightarrow \mathbb{R}$ is such that $\theta \circ \Gamma \in \mathcal{H}_{\delta}$.

Note that in these cases $\mathcal{L}$ acts only on the first variable of $K(\cdot, \zeta)$ and $\theta(\Gamma(\cdot, \zeta))$. 
Definition 2.2 Let $\mathcal{L}, K, \mathcal{H}_{0}$ be as above. Then we define the intervention operators $\mathcal{M}: \mathcal{H}_{0} \rightarrow \mathcal{H}_{0}$ and $\mathcal{M}_{\mathcal{L}}: \mathcal{H}_{0} \rightarrow \mathcal{H}_{0}$ as follows:

$$
\begin{aligned}
& \mathcal{M} h(y)=\sup _{\zeta \in Z}\{h(\Gamma(y, \zeta))+K(y, \zeta)\} ; \quad h \in \mathcal{H}_{0} \\
& \mathcal{M}_{\mathcal{L}} h(y)=\sup _{\zeta \in Z}\{h(\Gamma(y, \zeta))+\mathcal{L} K(y, \zeta)\} ; \quad h \in \mathcal{H}_{0} .
\end{aligned}
$$

We will also need the following concept:

Definition 2.3 Let $Y^{y}(t)$ be the state process without interventions and with starting point $Y^{y}(0)=y \in \mathbb{R}^{k}$. Let $\Gamma: \mathbb{R}^{k} \times \mathcal{Z} \rightarrow \mathbb{R}$ be the intervention function (see (1.2)). We say that $Y$ is $\Gamma$-homogeneous if

$$
\Gamma\left(Y^{y}(t), \zeta\right)=Y^{\Gamma(y, \zeta)}(t) \quad \text { for all } t \geq 0, y \in \mathbb{R}^{k}, \zeta \in \mathcal{Z} .
$$

Example 2.4 (i) If

$$
Y^{y}(t)=y+\eta(t)
$$

where $\eta$ is a $k$-dimensional Lévy process and

$$
\Gamma(y, \zeta)=y+\zeta ; \quad y \in \mathbb{R}^{k}, \quad \zeta \in \mathbb{R}^{k}
$$

then (??) holds, because

$$
\Gamma\left(Y^{y}(t), \zeta\right)=Y^{y}(t)+\zeta=y+\zeta+\eta(t)=\Gamma(y, \zeta)+\eta(t)=Y^{\Gamma(y, \zeta)}(t) .
$$

(ii) Similarly, if

$$
\Gamma(y, \zeta)=y \zeta ; \quad y, \zeta \in(0, \infty)
$$

and

$$
\left\{\begin{array}{l}
d Y^{y}(t)=Y^{y}\left(t^{-}\right)\left[\mu(t) d t+\sigma(t) d B(t)+\int_{\mathbb{R}} \gamma(t, z) \tilde{N}(d t, d z)\right] \\
Y^{y}(0)=y \in \mathbb{R}
\end{array}\right.
$$

where the processes $\mu(t), \sigma(t)$ and $\gamma(t, z)>-1$ do not depend on $Y^{y}(t)$, then (??) holds also. To see this, note that in this case we have

$$
Y(t)=y \exp R(t)
$$


where

$$
\begin{aligned}
R(t)=\int_{0}^{t}\left\{\mu(s)-\frac{1}{2} \sigma^{2}(s)+\int_{\mathbb{R}}(\log (1+\gamma(s, z))-\gamma(s, z)) \nu(d z)\right\} d s \\
\quad+\int_{0}^{t} \sigma(s) d B(s)+\int_{\mathbb{R}} \log (1+\gamma(s, z)) \tilde{N}(d s, d z) .
\end{aligned}
$$

Hence

$$
\begin{aligned}
\Gamma\left(Y^{y}(t), \zeta\right) & =Y^{y}(t) \zeta=y \zeta \exp R(t)=\Gamma(y, \zeta) \exp R(t) \\
& =Y^{\Gamma(y, \zeta)}(t), \quad \text { as claimed. }
\end{aligned}
$$

Lemma 2.5 Suppose the process $Y^{y}(t)$ is $\Gamma$-homogeneous. Let $\mathcal{M}$ and $\mathcal{M}_{\mathcal{L}}$ be as in Definition ??. Then

$$
\mathcal{L}(\mathcal{M} h)(y)=\mathcal{M}_{\mathcal{L}}(\mathcal{L} h)(y) ; \quad h \in \mathcal{H}_{0}, \quad y \in \mathbb{R}^{k} .
$$

Proof. For $\zeta \in \mathcal{Z}$ we have

$$
\begin{aligned}
\mathcal{L}(\mathcal{M} h)(y) & =\mathcal{L}\left(\sup _{\zeta \in \mathcal{Z}}\{h(\Gamma(y, \zeta))+K(y, \zeta)\}\right)(y) \\
& =\sup _{\zeta \in \mathcal{Z}}\{\mathcal{L}(h(\Gamma(\cdot, \zeta))+K(\cdot, \zeta))(y)\} \\
& =\sup _{\zeta \in \mathcal{Z}}\left\{E^{y}[h(\Gamma(Y(\delta), \zeta)]+\mathcal{L} K(y, \zeta)\}\right. \\
& =\sup _{\zeta \in \mathcal{Z}}\left\{E\left[h\left(\Gamma\left(Y^{y}(\delta), \zeta\right)\right)\right]+\mathcal{L} K(y, \zeta)\right\} \\
& =\sup _{\zeta \in \mathcal{Z}}\left\{E\left[h\left(Y^{\Gamma(y, \zeta)}(\delta)\right)\right]+\mathcal{L} K(y, \zeta)\right\} \\
& =\sup _{\zeta \in \mathcal{Z}}\left\{E^{\Gamma(y, \zeta)}[h(Y(\delta))]+\mathcal{L} K(y, \zeta)\right\} \\
& =\sup _{\zeta \in \mathcal{Z}}\{\mathcal{L} h(\Gamma(y, \zeta))+\mathcal{L} K(y, \zeta)\}=\mathcal{M}_{\mathcal{L}}(\mathcal{L} h(y)) .
\end{aligned}
$$

Lemma 2.6 Suppose $Y$ is $\Gamma$-homogeneous. Then

$$
\mathcal{L}(\theta \circ \Gamma)(y, \zeta)=(\mathcal{L} \theta) \circ \Gamma(y, \zeta)
$$

for all $\theta \in \mathcal{H}_{\delta}, \zeta \in \mathcal{Z}$ and $y \in \mathbb{R}^{k}$. 
Proof. By (2.1) and (2.6) we have

$$
\begin{aligned}
\mathcal{L}(\theta \circ \Gamma)(y, \zeta) & =E^{y}[\theta(\Gamma(Y(\delta), \zeta))]=E\left[\theta\left(\Gamma\left(Y^{y}(\delta), \zeta\right)\right)\right] \\
& =E\left[\theta\left(Y^{\Gamma(y, \zeta)}(\delta)\right)\right]=E^{\Gamma(y, \zeta)}[\theta(Y(\delta))]=(\mathcal{L} \theta)(\Gamma(y, \zeta)) .
\end{aligned}
$$

We now consider the case when at most $n$ interventions are allowed, as described in Problem ??. Define a sequence of delayed reaction optimal stopping problems as follows:

$$
\theta_{0}(y)=E^{y}\left[\int_{0}^{\tau_{\mathcal{S}}+\delta} f(Y(t)) d t+g\left(Y\left(\tau_{\mathcal{S}}+\delta\right)\right)\right]
$$

and inductively, for $j=1, \ldots, n$,

$$
\theta_{j}(y)=\sup _{\tau \in \mathcal{T}_{0}} E^{y}\left[\int_{0}^{\tau+\delta} f(Y(t)) d t+\mathcal{M} \theta_{j-1}(Y(\tau+\delta))\right] .
$$

Here $Y(t)$ is the process defined in (??), i.e. without interventions. Then, similar to the no-delay case, we have the following result:

Theorem 2.7 Suppose that

$$
Y(\cdot) \text { is } \Gamma \text {-homogeneous. }
$$

Moreover, suppose that for $j=1, \ldots, n$ there exists an optimal stopping time $\hat{\tau}_{j}$ for the delay optimal stopping problem (??) for $\theta_{j}$. Moreover, suppose that for all $j=1, \ldots, n$ and all $y$ there exists

$$
\bar{\zeta}_{j}(y) \in \operatorname{Argmax}\left\{\mathcal{L} \theta_{j-1}(\Gamma(y, \zeta))+\mathcal{L} K(y, \zeta) ; \zeta \in \mathcal{Z}\right\}
$$

and that the map

$$
y \rightarrow \bar{\zeta}_{j}(y) \text { has a measurable selection. }
$$

Then

$$
\theta_{n}(y)=\Phi_{\delta}^{(n)}(y) ; \quad y \in \mathcal{S}
$$


and the delayed reaction impulse control

$$
\hat{v}:=\left(\hat{\tau}_{1}+\delta, \ldots, \hat{\tau}_{n}+\delta ; \hat{\zeta}_{1}, \ldots, \hat{\zeta}_{n}\right)
$$

is optimal, where

$$
\hat{\zeta}_{j}=\bar{\zeta}_{j}\left(Y\left(\hat{\tau}_{j}\right)\right) ; \quad 1 \leq j \leq n
$$

ProOF. This follows by induction on $n$, combined with the strong Markov property. The details are as follows:

$n=1:$ First assume there is only one intervention allowed. If we decide at time $\tau \in \mathcal{T}_{0}$ to make an intervention of size $\zeta \in \mathcal{Z}$, then the intervention is carried out at time $\tau+\delta$. The corresponding state process is denoted by $Y^{(v)}(t)$, where $v=(\tau+\delta, \zeta)$. Note that $Y^{(v)}(t)=Y(t)$ for $0 \leq t \leq(\tau+\delta)^{-}$ and $Y^{(v)}(\tau+\delta)=\Gamma(Y(\tau+\delta))=\Gamma\left(\check{Y}^{(v)}\left((\tau+\delta)^{-}\right)\right)$. Hence by (1.13) and the strong Markov property

$$
\begin{aligned}
\Phi_{\delta}^{(1)}(y)= & \sup _{\tau, \zeta} E^{y}\left[\int_{0}^{\tau_{\mathcal{S}}+\delta} f\left(Y^{(v)}(t)\right) d t+g\left(Y^{(v)}\left(\tau_{\mathcal{S}}+\delta\right)\right)+K\left(\check{Y}^{(v)}\left((\tau+\delta)^{-}\right), \zeta\right)\right] \\
= & \sup _{\tau, \zeta} E^{y}\left[\int_{0}^{\tau+\delta} f(Y(t)) d t+\int_{\tau+\delta}^{\tau_{\mathcal{S}}+\delta} f\left(Y^{(v)}(t)\right) d t+g\left(Y^{(v)}\left(\tau_{\mathcal{S}}+\delta\right)\right)\right. \\
& +K(Y(\tau+\delta), \zeta)] \\
= & \sup _{\tau, \zeta} E^{y}\left[\int_{0}^{\tau+\delta} f(Y(t)) d t+E^{Y^{(v)}\left(\tau_{\mathcal{S}}+\delta\right)}\left[\int_{0}^{\tau_{\mathcal{S}}+\delta} f(Y(t)) d t+g\left(Y\left(\tau_{\mathcal{S}}+\delta\right)\right)\right]\right. \\
+ & K(Y(\tau+\delta), \zeta] \\
= & \sup _{\tau, \zeta} E^{y}\left[\int_{0}^{\tau+\delta} f(Y(t)) d t+\theta_{0}\left(Y^{(v)}(\tau+\delta)\right)+K(Y(\tau+\delta), \zeta)\right]
\end{aligned}
$$

$$
\leq \sup _{\tau} E^{y}\left[\int_{0}^{\tau+\delta} f(Y(t)) d t+\mathcal{M} \theta_{0}(Y(\tau+\delta))\right] .
$$


Here equality holds if we chose $\zeta=\hat{\zeta}_{1}$ such that

$$
E^{y}\left[\theta_{0}\left(\Gamma\left(Y(\tau+\delta), \hat{\zeta}_{1}\right)+K\left(Y(\tau+\delta), \hat{\zeta}_{1}\right)-\mathcal{M} \theta_{0}(Y(\tau+\delta))\right]=0,\right.
$$

i.e.

$$
E^{y}\left[E^{Y(\tau)}\left[\theta_{0}\left(\Gamma(Y(\delta)), \hat{\zeta}_{1}\right)+K\left(Y(\delta), \hat{\zeta}_{1}\right)-\mathcal{M} \theta_{0}(Y(\delta))\right]=0\right.
$$

or

$$
E^{y}\left[\mathcal{L}\left(\theta_{0} \circ \Gamma\right)\left(Y(\tau), \hat{\zeta}_{1}\right)+\mathcal{L} K\left(Y(\tau), \hat{\zeta}_{1}\right)-\mathcal{L}\left(\mathcal{M} \theta_{0}\right)(Y(\tau))\right]=0 .
$$

To achieve this it suffices to choose $\hat{\zeta}_{1}=\bar{\zeta}_{1}(Y(\tau))$, where $\bar{\zeta}_{1}=\bar{\zeta}_{1}(y)$ satisfies the equation

$$
\mathcal{L}\left(\theta_{0} \circ \Gamma\right)\left(y, \bar{\zeta}_{1}\right)+\mathcal{L} K\left(y, \bar{\zeta}_{1}\right)-\mathcal{L}\left(\mathcal{M} \theta_{0}\right)(y)=0 ; \quad y \in \mathcal{S}
$$

By (??) and (??) this is equivalent to

$$
\mathcal{L} \theta_{0}\left(\Gamma\left(y, \bar{\zeta}_{1}\right)\right)+\mathcal{L} K\left(y, \bar{\zeta}_{1}\right)-\mathcal{M}_{\mathcal{L}}\left(\mathcal{L} \theta_{0}\right)(y)=0 ; \quad y \in \mathcal{S} .
$$

This is achieved by (??), because by Definition ??, (??), applied to $h=\mathcal{L} \theta_{0}$ we have

$$
\mathcal{M}_{\mathcal{L}}\left(\mathcal{L} \theta_{0}\right)(y)=\sup _{\zeta \in \mathcal{Z}}\left\{\mathcal{L} \theta_{0}(\Gamma(y, \zeta))+\mathcal{L} K(y, \zeta) ; \zeta \in \mathcal{Z}\right\}
$$

This proves that with this choice $\hat{\zeta}_{1}=\bar{\zeta}_{1}(Y(\tau))$ equality holds in (??) and hence the theorem is proved for $n=1$.

$n>1$ : The proof in this case is similar to the case $n=1$. For $v=\left(\tau_{1}+\delta\right.$, $\left.\ldots, \tau_{n}+\delta ; \zeta_{1}, \ldots, \zeta_{n}\right) \in \mathcal{V}_{\delta}^{(n)}$ we put $\hat{v}=\left(\tau_{1}+\delta, \ldots, \tau_{n}+\delta ; \zeta_{2}, \ldots, \zeta_{n}\right) \in$ $\mathcal{V}_{\delta}^{(n-1)}$. By the induction hypothesis $\Phi_{\delta}^{(n-1)}(y)=\Theta_{n-1}(y)$. Hence by the dynamic programming principle (see e.g. [?], Prop. 3.2) we get 


$$
\begin{aligned}
& \Phi_{\delta}^{(j)}(y)=\sup _{v \in \mathcal{V}_{\delta}^{(n)}} E^{y}\left[\int _ { 0 } ^ { \tau _ { \mathcal { S } } + \delta } f \left(Y^{(v)}(t) d t+g\left(Y^{(v)}\left(\tau_{\mathcal{S}}+\delta\right)\right)\right.\right. \\
& \quad+\sum_{i=1}^{n} K\left(\check{Y}^{(v)}\left(\left(\tau_{i}+\delta\right)^{-}\right), \zeta_{i}\right] \\
& =\sup _{\tau_{1}, \zeta_{1}, \hat{v}} E^{y}\left[\int_{0}^{\tau_{1}+\delta} f(Y(t)) d t+K\left(Y\left(\tau_{1}+\delta\right), \zeta_{1}\right)+\Theta_{n-1}\left(Y^{(\hat{v})}\left(\tau_{1}+\delta\right)\right)\right] \\
& \leq \sup _{\tau_{1}} E^{y}\left[\int_{0}^{\tau_{1}+\delta} f(Y(t)) d t+\mathcal{M} \Theta_{n-1}\left(Y\left(\tau_{1}+\delta\right)\right)\right] .
\end{aligned}
$$

From here on the proof proceeds as in the case $n=1$. We omit the details.

In [?] it is proved that any delay optimal stopping problem can be transformed to a no-delay optimal stopping problem (without assuming $\Gamma$-homogeneity), as follows:

$$
\begin{aligned}
\sup _{\tau \in \mathcal{T}_{0}} E^{y}\left[\int_{0}^{\tau+\delta} f(Y(t)) d t+g(Y(\tau+\delta))\right] \\
=\sup _{\tau \in \mathcal{T}_{0}} E^{y}\left[\int_{0}^{\tau} f(Y(t)) d t+\left(\mathcal{L} g+F_{\delta}\right)(Y(\tau))\right]
\end{aligned}
$$

where

$$
F_{\delta}(y)=E^{y}\left[\int_{0}^{\delta} f(Y(t)) d t\right] .
$$


Using this we see that the iterated delay optimal stopping problems (??)-(??) are equivalent to the following no-delay iterated optimal stopping problems:

$$
\begin{array}{r}
\theta_{0}(y)=E^{y}\left[\int_{0}^{\tau_{\mathcal{S}}} f(Y(t)) d t+\left(\mathcal{L} g+F_{\delta}\right)\left(Y\left(\tau_{\mathcal{S}}\right)\right)\right] \\
\theta_{j}(y)=\sup _{\tau \in \mathcal{T}_{0}} E^{y}\left[\int_{0}^{\tau} f(Y(t)) d t+\left(\mathcal{L}\left(\mathcal{M} \theta_{j-1}\right)+F_{\delta}\right)(Y(\tau))\right] \\
\text { for } j=1,2, \ldots, n .
\end{array}
$$

\section{The general case}

In the case when there are no bounds on the number of interventions, we have the following result:

Theorem 3.1 Suppose the conditions of Theorem ?? hold. Define $\theta_{j}(y)$; $j=1,2, \ldots$ by the iterative procedure (??)-(??) or, equivalently, (??)-(??).

(i) Then

$$
\theta_{j}(y) \rightarrow \Phi_{\delta}(y) \quad \text { as } j \rightarrow \infty .
$$

(ii) Moreover, $\Phi_{\delta}(y)$ is a solution of the following non-linear delay optimal stopping problem

$$
\Phi_{\delta}(y)=\sup _{\tau \in \mathcal{T}_{0}} E^{y}\left[\int_{0}^{\tau+\delta} f(Y(t)) d t+\mathcal{M} \Phi_{\delta}(Y(\tau+\delta))\right]
$$

Proof. This is proved in [?] in the case of no delay. The same proof works in the delay case, in view of Theorem ??.

In view of (??) we also have

Theorem 3.2 Suppose the conditions of Theorem ?? hold. Then $\Phi_{\delta}(y)$ is a solution of the following non-linear, no-delay optimal stopping problem:

$$
\Phi_{\delta}(y)=\sup _{\tau \in \mathcal{T}_{0}} E^{y}\left[\int_{0}^{\tau} f(Y(t)) d t+\left(\mathcal{L}\left(\mathcal{M} \Phi_{\delta}\right)+F_{\delta}\right)(Y(\tau))\right]
$$


where, as before,

$$
F_{\delta}(y)=E^{y}\left[\int_{0}^{\delta} f(Y(t)) d t\right]
$$

Remark To the best of our knowledge general existence and uniqueness results for non-linear optimal stopping problems of this form are not known. We will not pursue this question here.

\section{An example}

We consider a delay version of Example 6.5 in [?]:

Suppose the difference $X^{(v)}(t)$ between the demand and the supply for a freight shipping company is modelled by

$$
\begin{cases}d X^{(v)}(t)=a d t+\sigma d B(t)+\int_{\mathbb{R}_{0}} z \tilde{N}(d t, d z) ; & \tau_{j}+\delta<t<\tau_{j+1}+\delta \\ (\text { where } a, \sigma \text { are constants and } z \leq 0 \text { a.s. } \nu) & \\ X^{(v)}\left(\tau_{j+1}+\delta\right)=\check{X}^{(v)}\left(\left(\tau_{j+1}+\delta\right)^{-}\right)-\zeta_{j+1} ; & j=0,1,2, \ldots \\ X^{(v)}\left(0^{-}\right)=x \in \mathbb{R} & \end{cases}
$$

Here $v=\left(\tau_{1}+\delta, \tau_{2}+\delta, \ldots ; \zeta_{1}, \zeta_{2}, \ldots\right)$ is a delay impulse control, $\tau_{j}$ represents time number $j$ when we decide to intervene by ordering more shipping capacity, and $\zeta_{j}$ denotes the additional capacity ordered. The cost of such an intervention at time $t$ is assumed to be

$$
K(t, \zeta)=e^{-\rho t}(c+\lambda \zeta)
$$

where $\rho>0, c>0$ and $\lambda \geq 0$ are constants. The expected total cost associated to such an impulse $v$ is assumed to be

$$
J^{(v)}(y)=E^{s, x}\left[\int_{0}^{\infty} e^{-\rho(s+t)}\left(X^{(v)}(t)\right)^{2} d t+\sum_{j=1}^{N} e^{-\rho\left(s+\tau_{j}+\delta\right)}\left(c+\lambda \zeta_{j}\right)\right],
$$

where $N$ is the number of interventions. Suppose only one intervention is allowed. Then the problem is to find $\Phi_{\delta}^{(1)}(y)$ and $v^{*} \in \mathcal{V}_{\delta}^{(1)}$ such that

$$
\Phi_{\delta}^{(1)}(y)=\sup _{v \in \mathcal{V}_{\delta}^{(1)}} J^{(v)}(y)=J^{\left(v^{*}\right)}(y), \quad \text { where } N=1 .
$$


To put this problem in the context of this paper we define

$$
Y^{(v)}(t)=\left[\begin{array}{c}
s+t \\
X^{(v)}(t)
\end{array}\right] \in \mathbb{R}^{2}, \quad y=\left(y_{1}, y_{2}\right)=(s, x)=Y^{(v)}(0)
$$

and put

$$
f(y)=f\left(y_{1}, y_{2}\right)=e^{-\rho y_{1}} y_{2}^{2}
$$

and

$$
K(y, \zeta)=e^{-\rho y_{1}}(c+\lambda \zeta), \quad m^{2}=\int_{\mathbb{R}_{0}} z^{2} \nu(d z) .
$$

Then

$$
\begin{aligned}
F_{\delta}(y) & =E^{y}\left[\int_{0}^{\delta} f(Y(t)) d t\right]=E^{y}\left[\int_{0}^{\delta} e^{-\rho(s+t)}(X(t))^{2} d t\right] \\
& =\int_{0}^{\delta} e^{-\rho(s+t)}\left[x^{2}+2 a x t+a^{2} t^{2}+\left(\sigma^{2}+m^{2}\right) t\right] d t \\
& =e^{-\rho s}\left[A x^{2}+C x+D\right],
\end{aligned}
$$

where

$$
A=A_{\delta}=\frac{1}{\rho}\left(1-e^{-\rho \delta}\right), \quad C=C_{\delta}=\frac{2 a}{\rho}\left[A_{\delta}-\delta e^{-\rho \delta}\right]
$$

and

$$
\begin{aligned}
D=D_{\delta}=\frac{1}{\rho}[ & \left(\sigma^{2}+m^{2}\right)\left(-\delta e^{-\rho \delta}+\frac{1}{\rho}\left(1-e^{-\rho \delta}\right)\right. \\
& \left.+a^{2}\left(-\delta^{2} e^{-\rho \delta}-\frac{2 \delta}{\rho} e^{-\rho \delta}+\frac{2}{\rho^{2}}\left(1-e^{-\rho \delta}\right)\right)\right] .
\end{aligned}
$$

By (??) we have

$$
\begin{aligned}
\theta_{0}(y) & =\int_{0}^{\infty} e^{-\rho(s+t)}\left[x^{2}+\left(2 a x+\sigma^{2}+m^{2}\right) t+a^{2} t^{2}\right] d t \\
& =e^{-\rho s}\left[\frac{1}{\rho} x^{2}+\left(2 a x+\sigma^{2}+m^{2}\right) \frac{1}{\rho^{2}}+2 a^{2} \frac{1}{\rho^{3}}\right] .
\end{aligned}
$$


Hence

$$
\begin{aligned}
\mathcal{M} \theta_{0}(y) & =\inf \left\{\theta_{0}(x-\zeta)+e^{-\rho s}(c+\lambda \zeta) ; \zeta \geq 0\right\} \\
& =\left\{\begin{array}{l}
e^{-\rho s}\left[\frac{\lambda^{2} \rho}{4}+c+\frac{\sigma^{2}+m^{2}}{\rho^{2}}+\frac{2 a^{2}}{\rho^{3}}\right] ; \quad x \geq \frac{\lambda \rho}{2}-\frac{a}{\rho} \\
e^{-\rho s}\left[\frac{1}{\rho} x^{2}+c+\frac{2 a x+\sigma^{2}+m^{2}}{\rho^{2}}+\frac{2 a^{2}}{\rho^{3}}\right] ; \quad x<\frac{\lambda \rho}{2}-\frac{a}{\rho}
\end{array}\right.
\end{aligned}
$$

which is attained at

$$
\zeta=\hat{\zeta}_{1}=\left(x+\frac{a}{\rho}-\frac{\lambda \rho}{2}\right)^{+}
$$

Put

$$
G(s, x)=e^{-\rho s}\left[\frac{1}{\rho}\left(x-\hat{\zeta}_{1}\right)^{2}+\frac{2 a}{\rho^{2}}\left(x-\hat{\zeta}_{1}\right)+\lambda \hat{\zeta}_{1}\right]
$$

Then

$$
\begin{aligned}
\mathcal{L}\left(\mathcal{M} \theta_{0}\right)(y) & =E^{y}\left[\mathcal{M} \theta_{0}(Y(\delta))\right] \\
& =E^{y}[G(Y(\delta))+M]=\mathcal{L} G(y)+M,
\end{aligned}
$$

where

$$
M=c+\frac{\sigma^{2}+m^{2}}{\rho^{2}}+\frac{2 a^{2}}{\rho^{3}} .
$$

Hence (??) gives the optimal stopping problem

$$
\begin{aligned}
\theta_{1}(y)=\inf _{\tau \in \mathcal{T}_{0}} E^{y}\left[\int_{0}^{\tau} e^{-\rho(s+t)} X^{2}(t) d t+\mathcal{L} G(Y(\tau))\right. \\
\left.+e^{-\rho(s+\tau)}\left(A X^{2}(\tau)+C X(\tau)+D\right)\right]+M .
\end{aligned}
$$

This is a classical optimal stopping problem, which can be solved by the usual method of variational inequalities. See e.g. [ØS, Chapter 2].

Our conclusion is the following, based on Theorem ?? and (??)-(??):

Theorem 4.1 Let $\tau^{*} \in \mathcal{T}_{0}$ be an optimal stopping time for the problem (??).

Define

$$
\hat{\tau}=\tau^{*}+\delta \quad \text { and } \quad \hat{\zeta}_{1}=\left(X\left(\tau^{*}\right)+\frac{a}{\rho}-\frac{\lambda \rho}{2}\right)^{+} .
$$

Then $\left(\hat{\tau}, \hat{\zeta}_{1}\right)$ is an optimal impulse control for the delayed reaction impulse control (??)-(??). 


\section{References}

[AK] L. Alvarez and J. Keppo: The impact of delivery lags on irreversible investment under uncertainty. European J. Operational Research 136 (2002), 173-180.

[BE] E. Bayraktar and M. Egami: The effects of implementation delay on decision-making under uncertainty. Stoch. Proc. and Their Applications (to appear).

[BS] A. Bar-Ilan and A. Sulem: Explicit solution of inventory problems with delivery lags. Math. Operations Research 20 (1995), 709-720.

[BL] A. Bensoussan and J. L. Lions: Impulse Control and Quasi-Variational Inequalities. Gauthier-Villars, Paris 1984.

[BP] B. Bruder and H. Pham: Impulse control problem on finite horizon with execution delay. Manuscript March 26, 2007.

[I] Y. Ishikawa: Optimal control problem associated with jump processes. Appl. Math. \& Optim. 50 (2004), 21-65.

[Ø] B. Øksendal: Optimal stopping with delayed information. Stochastics and Dynamics 5 (2005), 271-280.

[ØS] B. Øksendal and A. Sulem: Applied Stochastic Control of Jump Diffusions. Second Edition. Springer 2007.

[P] H. Pham: Optimal stopping of controlled jump diffusion processes: A viscosity solution approach. J. Math. Systems, Estimation, and Control 8 (1998), 1-27. 